[23] P. Lin and Y. M. Jia, "Average consensus in networks of multi-agents with both switching topology and coupling time-delay," Physica A, vol. 387, pp. 303-313, 2008.

[24] Y. P. Tian and C. L. Liu, "Consensus of multi-agent systems with diverse input and communication delays," IEEE Trans. Autom. Control, vol. 53 , no. 9 , pp. 2122-2128, Sep. 2008.

[25] F. Xiao and L. Wang, "Consensus protocols for discrete-time multiagent systems with time-varying delays," Automatica, vol. 44, no. 10, pp. 2577-2582, 2008 .

[26] W. L. Lu, T. P. Chen, and G. R. Chen, "Synchronization analysis of linearly coupled systems described by differential equations with a coupling delay," Physica D, vol. 221, pp. 118-134, 2006

[27] T. A. Burton, Stability and Periodic Solutions of Ordinary and Functional Differential Equations. Orlando, FL: Academic Press, 1985.

[28] H. Yoshiyuki, M. Satoru, and N. Toshiki, Functional Differential Equations With Infinite Delay. New York: Springer-Verlag, 1991.

[29] T. P. Chen and L. L. Wang, "Global $\mu$-stability of delayed neural networks with unbounded time-varying delays," IEEE Trans. Neural Netw., vol. 18, no. 6, pp. 1836-1840, Jun. 2007.

[30] T. P. Chen and L. L. Wang, "Power-rate global stability of dynamical systems with unbounded time-varying delays," IEEE Trans. Circuits Syst. II, vol. 54, no. 8, pp. 705-709, Aug. 2007.

[31] C. W. Wu and L. O. Chua, "Synchronization in an array of linearly coupled dynamical systems," IEEE Trans. Circuits Syst. I, vol. 42, no. 8, pp. 430-447, Aug. 1995.

[32] J. Hale, Theory of Functional Differential Equations. New York: Springer-Verlag, 2003.

\section{Manufacturing Systems With a Production Dependent Failure Rate: Structure of Optimality}

Francesco Martinelli

\begin{abstract}
This technical note provides the structure of a policy minimizing a long term, average, expected, backlog/inventory cost for a fluid model, single machine, single product manufacturing system subject to a failure/repair Markov process, where the failure rate is a piecewise constant function of the production rate. This policy generalizes previous results and confirms several conjectures reported in the literature, providing an interesting insight into the problem.
\end{abstract}

Index Terms-Markov processes, production dependent failure rates, threshold policies, unreliable systems.

\section{INTRODUCTION}

A large literature deals with the problem of failure prone manufacturing systems. A complete analytical solution has been given in [1] for a single machine characterized by a homogeneous Markov failure/repair process. In such case the control minimizing a long term average expected cost penalizing both surplus and backlog is the hedging point policy, according to which the machine is operated at full rate until the inventory level hits a non-negative hedging level (or safety stock) $Z$.

Manuscript received March 19, 2008; revised February 23, 2009, January 18, 2010, and June 10, 2010; accepted June 18, 2010. Date of publication June 28, 2010; date of current version October 06, 2010. This work was supported in part by MIUR under Grants PRIN 2005092439 and 2007ZMZK5T. Recommended by Associate Editor X. Xie.

The author is with the Dipartimento di Informatica, Sistemi e Produzione, Università di Roma "Tor Vergata," Rome I-00133, Italy (e-mail: martinelli@disp.uniroma2.it).

Digital Object Identifier 10.1109/TAC.2010.2054790
The problem becomes much more involved if the failure rate depends on the production rate. In [3] it has been proved that the hedging point policy remains optimal if and only if the dependence of the failure rate on the production rate is affine and it was conjectured for more general cases, e.g., when this dependence is quadratic, that as the inventory level approaches a "hedging level," it may be beneficial to decrease the production rate to gain in reliability. This conjecture was actually confirmed by the numerical results reported in [4].

An analytical increment in this direction, still confirming the conjecture in [3], was presented in [5], where it was considered a machine characterized by two failure rates: one for low and one for high production rates. In this technical note we generalize this problem by considering a machine with $N$ different failure rates: more specifically, the failure rate is assumed to depend on the production rate through an increasing, piecewise constant function. This makes the proof of optimality much more involved with respect to the one given in [5] since it has not been possible in this case to derive in closed form several parameters characterizing the optimal control.

Sufficient conditions for the existence of an optimal control are given in this note. The optimal policy, which is a multi-level, decreasing, piecewise constant, feedback function of the backlog/inventory level, allows to obtain the following interesting insight: the production rates providing the maximum expected long run buffer increment are convenient when the backlog/inventory level is far from the safety stock, while the production rates guaranteeing the maximum expected up times are better when approaching the safety stock. The shape of the optimal policy strongly depends on the convexity properties of the failure rate function, confirming also in this case the numerical findings of [4] and the analytical results of [3].

\section{NOTATION AND PROBLEM FORMULATION}

Let $x(t)$ denote the buffer content at time $t$, with $x(t)>0$ representing an inventory surplus and $x(t)<0$ a backlog of $-x(t)$. Let $d$ be the constant demand rate to be met. Then the buffer level $x(t)$ at time $t$ satisfies the following dynamical equation:

$$
\dot{x}=u(t)-d
$$

where the production rate $u(t)=0$ if at time $t$ the machine is in the down state (also referred to as state 0 ), and $u(t) \in[0, \mu]$ if at time $t$ the machine is in the up state (also referred to as state 1 ). We assume a Markov failure/repair process: the repair rate $q_{u p}$ is constant while the failure rate $q_{d}(u)$ depends on the production rate $u$ as follows:

$$
q_{d}(u):= \begin{cases}q_{1} & u \leq U_{1} \\ q_{2} & u \in\left(U_{1}, U_{2}\right] \\ \cdots & \\ q_{N} & u \in\left(U_{N-1}, U_{N}\right]\end{cases}
$$

where $0<q_{1}<q_{2}<\ldots<q_{N}$ and $0<U_{1}<U_{2}<\ldots<$ $U_{N}=: \mu$. The piecewise constant function in (2) may result from the discrete approximation of a continuous function, for example of the type considered in [3]

$$
Q_{d}(u)=a u^{\beta}+b
$$

with $a, b$ and $\beta$ non-negative constants. The state of the machine at time $t$ will be denoted by $s(t)$, hence $s(t) \in\{0,1\}$ for all $t$. The scheduling problem considered in this technical note is the 
determination of the optimal control $u^{*}(t)$ minimizing the long-term average expected cost

$$
J=\limsup _{T \rightarrow \infty} \frac{1}{T} E\left[\int_{0}^{T} g[x(t)] d t\right]
$$

where $g(x)=c_{p} x^{+}+c_{m} x^{-}$, with $x^{-}=\max \{0,-x\}, x^{+}=$ $\max \{0, x\}, c_{p}$ and $c_{m}$ non-negative constants. We are interested only in admissible control laws, i.e., in non anticipative policies such that for all $t \geq 0,0 \leq u(t) \leq \mu \cdot s(t)$ (see, e.g., [7] for more details on this). If the machine is operated at rate $u \in[0, \mu]$, the average production rate at steady state is $u q_{u p} /\left(q_{u p}+q_{d}(u)\right)$. The following feasibility assumption will be considered, that there exists at least one $U_{i}$ such that the demand may be met in the average operating the machine at $U_{i}$. If this assumption were not satisfied, any rate $u \in[0, \mu]$ would be unfeasible and the backlog, under any policy, would increase with no bound over time.

Assumption 1: Let

$$
\Delta_{i}:=U_{i} q_{u p}-d\left(q_{u p}+q_{i}\right) .
$$

Then, $\Delta_{i}>0$ for at least one $i \in\{1, \ldots, N\}$.

If, for some $i, \Delta_{i}>0$, then also $U_{i}>d$. So, for each $i$, the quantity $\alpha_{i}:=\Delta_{i} / d\left(U_{i}-d\right)$ (used below) is positive if $U_{i}$ is feasible (i.e., if $\left.\Delta_{i}>0\right)$.

\section{The Structure OF THE Optimal POlicy}

The optimal policy for the considered problem has the following structure:

$$
u(x):= \begin{cases}0 & x>X_{\ell} \\ d & x=X_{\ell} \\ U_{i_{\ell}} & x \in\left[X_{\ell+1}, X_{\ell}\right) \\ U_{i_{\ell+1}} & x \in\left[X_{\ell+2}, X_{\ell+1}\right) \\ \cdots & \\ U_{i_{L}} & x<X_{L}\end{cases}
$$

with $1 \leq \ell \leq L \leq N$ and $\mathcal{S}:=\left\{i_{k}\right\}_{k=\ell, \ldots, L}, 1 \leq i_{\ell}<i_{\ell+1}<$ $\ldots<i_{L} \leq N$, the sequence of integers derived with the procedure reported in Algorithm 1 below (the reason behind the fact that the first element of $\mathcal{S}$ is denoted by $\ell$ and not by 1 will be explained below). Notice that $Z:=X_{\ell}$ in (6) is the hedging level, i.e., the safety stock to be maintained by the system, while all the $X_{i}$ 's, for $i>\ell$, are only thresholds where the production rate changes.

The procedure to derive the sequence $\mathcal{S}$ appearing in (6), reported below in Algorithm 1, requires the definition of the following positive quantities:

$$
\phi_{i j}=\frac{q_{j}-q_{i}}{U_{j}-U_{i}}
$$

for $1 \leq i<j \leq N$. Also, we need to define, for any $j, l \in\{1, \ldots, N\}$

$$
\Delta U_{j, l}:=\left(q_{u p}+q_{l}\right) U_{j}-\left(q_{u p}+q_{j}\right) U_{l}
$$

Algorithm 1: Generate at first the following sequence $\mathcal{S}^{\prime}=$ $\left\{i_{k}\right\}_{k=1, \ldots, L^{\prime}}$ of indexes:

$$
i_{k}= \begin{cases}1 & k=1 \\ \arg \min _{j>i_{k-1}} \phi_{i_{k-1}, j} & k=2, \ldots, L^{\prime}\end{cases}
$$

where $L^{\prime} \leq N$ is the first index for which $i_{L^{\prime}}=N$. Then the sequence $\mathcal{S}$ is given by the elements $\left\{i_{\ell}, i_{\ell+1}, \ldots, i_{L}\right\}$ of $\mathcal{S}^{\prime}$, where $i_{\ell}$ is the first index such that $U_{i_{\ell}}>d$ (under Assumption 1 it can be shown that such an $i_{\ell}$ is always comprised in $\mathcal{S}^{\prime}$ ) and $L \geq \ell$ is the first index (if any) for which it happens that $\Delta U_{i_{L}, i_{L+1}} \geq 0$, while $L=L^{\prime}$ if $\Delta U_{i_{k}, i_{k+1}}<0$ for all $k=\ell, \ldots, L^{\prime}-1$.

When using a policy in the class reported in (6), the cost index in (4) is a function of the levels $X_{k}$ 's, i.e., $J=J\left(X_{\ell}, \ldots, X_{L}\right)$ and we will denote by $X_{k}^{*}$, the optimal value of $X_{k}$, and by $J^{*}$ the corresponding optimal cost, i.e.

$$
J^{*}=J\left(X_{\ell}^{*}, \ldots, X_{L}^{*}\right)=\min _{X_{\ell} \geq \ldots \geq X_{L}} J\left(X_{\ell}, \ldots, X_{L}\right) .
$$

The optimal value of the $X_{k}$ 's can be numerically derived as illustrated in [6], where the analytical expression of $J\left(X_{\ell}, \ldots, X_{L}\right)$, for any $X_{\ell} \geq \ldots \geq X_{L}$, is given. It must be remarked that the optimal hedging level $Z^{*}=X_{\ell}^{*}$ can not be negative, as shown in [6].

\section{A. General Observations and Some Particular Cases}

The optimal policy in (6) operates the machine by selecting for each buffer level the production rate providing the best trade off between expected long run buffer increment and expected uptime, giving more importance to the former when the inventory level is far from $Z^{*}$ and to the latter when approaching the safety stock. In fact, the sequence of the expected uptimes $1 / q_{i_{k}}, k=\ell, \ldots, L$, associated with the production rates in $\mathcal{S}$, is decreasing with $k$ (since $U_{i_{k}}<U_{i_{k+1}}$ for all $k$ ) and the sequence of the expected long run buffer increments $E_{i_{k}}:=q_{u p} U_{i_{k}} /\left(q_{u p}+q_{i_{k}}\right)$ associated with the $U_{i_{k}}$ is increasing with $k$ (indeed, for all $i_{k} \in \mathcal{S}, \Delta U_{i_{k}, i_{k+1}}<0$ and, as it is straightforward to verify, $\Delta U_{j, l}>0$ if and only if $E_{j}>E_{l}$ ). This aligns with the conjecture reported in [3] where it is remarked how the optimal policy decreases the production rate approaching the hedging level to gain in reliability. Actually, based on the results of this technical note, we can say that, when approaching the hedging level, the optimal policy, to gain in reliability, decreases not simply the production rate but the effective production rate.

Another interesting observation concerns a major difference (described also in [4] based on numerical results) arising in the optimal policy between the case the failure rate $q_{d}(u)$ is a convex function of the production rate and the case it is affine or concave. If $q_{d}(u)$ is convex (i.e. $\phi_{i, i+1}<\phi_{i+1, i+2}$ for all $i=1, \ldots, N-2$-this happens if discretizing a convex function like the one in (3) when $\beta>1$ ), the procedure above gives $L^{\prime}=N, \mathcal{S}^{\prime}=\{1,2, \ldots, N\}$ and in general $L \leq L^{\prime}$, depending on the steepness of the function $q_{d}(u)$. This aligns with the numerical results of [4] where it was observed how, in the convex case, the production rate is smoothly decreased when approaching the safety stock. If $Q_{d}(u)$ is affine, any discretization would provide $\phi_{i, i+1}=\phi_{i+1, i+2}$ for all $i=1, \ldots, N-2$. The results of this technical note align then with the analytical findings of [3] according to which, in the affine case, the optimal policy is the hedging point policy. In fact, in this case, we obtain $L^{\prime}=2, \mathcal{S}^{\prime}=\{1, N\}$ and, if $U_{1}<d, \ell=L=2$ and $\mathcal{S}=\{N\}$, which corresponds to the hedging point policy since the production rate is sharply reduced from the maximum production rate $U_{N}$ to 0 . Finally, if the $q_{d}(u)$ is concave (i.e. $\phi_{i, i+1}>\phi_{i+1, i+2}$ for all $i=1, \ldots, N-2$-this happens if discretizing a $Q_{d}(u)$ in (3) when $\beta<1$ ), we still obtain $L^{\prime}=2$, $\mathcal{S}^{\prime}=\{1, N\}$ and, if $U_{1}<d, \ell=L=2$ and $\mathcal{S}=\{N\}$. This seems to correspond, as in the affine case, to a hedging point policy. However now the obtained policy does not satisfy the optimality conditions reported below in Theorem 2 (see Remark 1). This also aligns with the numerical findings of [4], where it was conjectured that the optimal policy in the concave case is only asymptotic, and consists of a hedging point policy where the safety stock is maintained through an infinite switching of the production rate between 0 and $\mu$. 


\section{PROOF OF Optimality}

As in [5], we use the following result to assess the optimality of (6) (with optimal levels $X_{i}$ 's). Its proof, under certain regularity and stability conditions imposed on the control (actually met, under Assumption 1 , by any policy in the class (6)) is essentially like the one given in [1].

Theorem 1: (Verification Theorem) If there exist a constant $J^{*}$ and two continuously differentiable functions $V(x, 0)$ and $V(x, 1)$, $|V(x, i)| \leq c_{1} x^{2}+c_{2}$ for some constants $c_{1}$ and $c_{2}(i=0,1)$, such that a control $u(x)$ satisfies the following HJB equations:

$$
\begin{aligned}
& \min _{u \in[0, \mu]}\left\{(u-d) d V(x, 1) / d x+q_{d}(u)[V(x, 0)-V(x, 1)]\right\} \\
& \quad=J^{*}-g(x) \\
& d \frac{d V(x, 0)}{d x}+q_{u p}[V(x, 0)-V(x, 1)] \\
& \quad=g(x)-J^{*}
\end{aligned}
$$

then $u(x)$ is optimal and $J^{*}$ is the optimal cost.

The following result states that, under Assumption 1, if some additional condition is met, the policy given in (6) (with suitable levels $X_{i}$ 's) is optimal.

Theorem 2: If Assumption 1 is satisfied together with one of the following two conditions:

i) $U_{i}>d$ for all $i$

ii) there exist $i_{j-1}, i_{j}, i_{j+1} \in \mathcal{S}^{\prime}$ such that $U_{i_{j}}=d$ and $\left(q_{i_{j+1}}-\right.$ $\left.q_{i_{j}}\right) /\left(U_{i_{j+1}}-U_{i_{j}}\right)=\left(q_{i_{j}}-q_{i_{j-1}}\right) /\left(U_{i_{j}}-U_{i_{j-1}}\right)$; the optimal policy has the structure reported in (6).

Remark 1: Condition (i) in the theorem agrees with the result reported in [5] for $N=2$ and also with the classical solution of the problem with a constant failure rate function $q_{d}(u)=q_{d}$, solved in [1]. Condition (ii) allows to explain several (analytical and numerical) results reported in the literature. In particular Condition (ii) is met if the considered $q_{d}(u)$ comes from the discretization of an affine failure rate function $Q_{d}(u)$ (if $U_{i}=d$ is selected in the discretization), which allows to understand the optimality of the hedging point policy for affine failure rate functions, as also proved in [3]. If $Q_{d}(u)$ is convex, it is always possible to meet Condition (ii) as the discretization step goes to zero around the value $u=d$. This would match with the numerical results reported in [4]. If the continuous failure rate function $Q_{d}(u)$ is concave, there is no way of satisfying Condition (ii) if $U_{1}<d$, and this would confirm the discussion in [4] where it was conjectured that an optimal feedback control does not exist in the concave case. Considering Condition (ii), and not simply that there exists $i_{j} \in \mathcal{S}^{\prime}$ such that $U_{i_{j}}=d$, allows to prove the continuity of $V_{x}(x, 1)$ needed in the Verification Theorem: this is identical to the reason reported in [8] for not including $d$ in the capacity set of the machine (Assumption A5 in [8]). The last equality in Condition (ii) ensures in fact that $d$ can be optimal only on a point and not over a finite interval (even if this interval would be a transient set). However, in [6], it has been shown through some numerical examples that Conditions (i) and (ii) of the theorem are not necessary for the optimality of (6) and it seems enough in Case (ii) the fact that $d$ belongs to $\mathcal{S}^{\prime}$.

To prove the theorem we need the following two lemmas. Lemma 1 states that the segments $\left(-\infty, \phi_{i_{1} i_{2}}\right),\left(\phi_{i_{1} i_{2}}, \phi_{i_{2} i_{3}}\right), \ldots$, $\left(\phi_{i_{L^{\prime}-1^{i} L^{\prime}}}, \infty\right)$, provide a partition of the real axis. In Lemma 2 , it is proved that, if some $i_{k} \in \mathcal{S}^{\prime}$ is more efficient than the next element $i_{k+1} \in \mathcal{S}^{\prime}$ (i.e., $\Delta U_{i_{k}, i_{k+1}}>0$ ) then this holds for all subsequent elements of $\mathcal{S}^{\prime}$.

Lemma 1: Let $q_{d}(u)$ as defined in (2), $\phi_{i j}$ as defined in (7) and consider the sequence $\mathcal{S}^{\prime}=\left\{i_{1}, \ldots, i_{L^{\prime}}\right\}$, defined in Section III. Then this sequence is such that

$$
i_{k}=\arg \min _{j<i_{k+1}}\left(-\phi_{j, i_{k+1}}\right)
$$

for all $k=1, \ldots, L^{\prime}-1$, hence

$$
\begin{aligned}
m_{k+1} & :=\min _{j<i_{k+1}}\left(-\phi_{j, i_{k+1}}\right) \\
& =-\phi_{i_{k}, i_{k+1}}=\max _{j>i_{k}}\left(-\phi_{i_{k}, j}\right)=: M_{k}
\end{aligned}
$$

for all $k=1, \ldots, L^{\prime}-1$, and the intervals

$$
\left(-\infty, m_{L^{\prime}}\right],\left[M_{L^{\prime}-1}, m_{L^{\prime}-1}\right],\left[M_{L^{\prime}-2}, m_{L^{\prime}-2}\right], \ldots,\left[M_{1}, \infty\right)
$$

provide a partition of the real axis and, for all $j \notin \mathcal{S}^{\prime}$

$$
\min _{i<j}\left(-\phi_{i, j}\right)<\max _{i>j}\left(-\phi_{j, i}\right) .
$$

Proof: The proof follows directly by observing that sequence $\mathcal{S}^{\prime}$ defines the convex envelop of function $q_{d}(u)$.

Lemma 2: Let $i_{k} \in \mathcal{S}^{\prime}$, with $k \leq L^{\prime}-2$. Then $\Delta U_{i_{k}, i_{k+1}} \geq 0$ implies $\Delta U_{i_{k+1}, i_{k+2}} \geq 0$.

Proof: From the definition of $\phi_{i j}$, it is possible to write

$$
q_{i_{k+2}}=q_{i_{k+1}}+\phi_{i_{k+1}, i_{k+2}}\left(U_{i_{k+2}}-U_{i_{k+1}}\right) .
$$

Substituting in the definition of $\Delta U_{i_{k+1}, i_{k+2}}$ we obtain

$$
\Delta U_{i_{k+1}, i_{k+2}}=\left(U_{i_{k+2}}-U_{i_{k+1}}\right)\left[\phi_{i_{k+1}, i_{k+2}} U_{i_{k+1}}-\left(q_{u p}+q_{i_{k+1}}\right)\right] \text {. }
$$

Similarly, it holds

$$
\Delta U_{i_{k}, i_{k+1}}=\left(U_{i_{k+1}}-U_{i_{k}}\right)\left[\phi_{i_{k}, i_{k+1}} U_{i_{k}}-\left(q_{u p}+q_{i_{k}}\right)\right] .
$$

Since we have that $\Delta U_{i_{k}, i_{k+1}} \geq 0$, the previous equation implies

$$
\left[\phi_{i_{k}, i_{k+1}} U_{i_{k}}-\left(q_{u p}+q_{i_{k}}\right)\right] \geq 0 .
$$

Applying the definition used in (15) at $k+1$, we obtain

$$
q_{i_{k+1}}=q_{i_{k}}+\phi_{i_{k}, i_{k+1}}\left(U_{i_{k+1}}-U_{i_{k}}\right)
$$

which, substituted in (16), gives

$$
\begin{array}{r}
\Delta U_{i_{k+1}, i_{k+2}}=\left(U_{i_{k+2}}-U_{i_{k+1}}\right)\left[\phi_{i_{k+1}, i_{k+2}} U_{i_{k+1}}-q_{u p}-q_{i_{k}}\right. \\
\left.-\phi_{i_{k}, i_{k+1}} U_{i_{k+1}}+\phi_{i_{k}, i_{k+1}} U_{i_{k}}\right]
\end{array}
$$

Using (18), (20) becomes

$$
\Delta U_{i_{k+1}, i_{k+2}} \geq\left(U_{i_{k+2}}-U_{i_{k+1}}\right)\left[\phi_{i_{k+1}, i_{k+2}}-\phi_{i_{k}, i_{k+1}}\right] U_{i_{k+1}}
$$

which is non negative, being $\phi_{i_{k+1}, i_{k+2}} \geq \phi_{i_{k}, i_{k+1}}$. In fact, by definition, $\phi_{i_{k}, i_{k+1}} \leq \phi_{i_{k}, j}$ for all $j>i_{k}$, in particular

$$
\phi_{i_{k}, i_{k+1}} \leq \phi_{i_{k}, i_{k+2}} .
$$

Similarly, $\phi_{i_{k+1}, i_{k+2}} \geq \phi_{j, i_{k+2}}$ for all $j<i_{k+2}$, hence

$$
\phi_{i_{k+1}, i_{k+2}} \geq \phi_{i_{k}, i_{k+2}} \text {. }
$$

From (22) and (23), it follows $\phi_{i_{k+1}, i_{k+2}} \geq \phi_{i_{k}, i_{k+1}}$.

Proof of Theorem 2: Let $v(x, 0)$ and $v(x, 1)$ denote the differential costs associated with the optimal policy starting from $x(0)=x$ with a down and with an up machine respectively (see, e.g., [10] for a definition of these functions). Following the procedure in Appendix $\mathrm{H}$ of [9], it is possible to show that $V(x, 0):=v(x, 0)+c$ and $V(x, 1):=$ $v(x, 1)+c$ (where $c$ is any constant) satisfy (10), (11) at least in the viscosity sense. Take for simplicity $c=0: V(x, i)$ will then represent from now on the differential costs associated with the optimal policy. Now, $V(x, 0)$ is continuously differentiable for all $x$ while $V(x, 1)$ may be not differentiable only on the switching levels, i.e. where the control is discontinuous (see e.g. [10], Section IV or [2], ch. 9.3). However, under Condition (i) or (ii) of the theorem, it is possible to prove (see [6]) that $V(x, 1)$ is differentiable also on the switching levels. This 
will imply that $V(x, 0)$ and $V(x, 1)$ are a classical $\left(\mathcal{C}^{1}\right)$ solution to $(10)$ and (11). Let:

$$
V_{x}(x, 1):=\frac{d V(x, 1)}{d x}, \quad H(x):=V(x, 0)-V(x, 1) .
$$

The proof of the theorem is based on the following properties: a) $H(x)>0$ for all $x$; b) $V(x, 0)$ and $V(x, 1)$ are $\mathcal{C}^{1}$ functions; c) $V(x, 0)$ and $V(x, 1)$ are quadratically bounded in modulus (i.e., as requested in the Verification Theorem, $|V(x, i)| \leq c_{1} x^{2}+c_{2}, i=0$, $1)$; and d) they are also bounded from below by a quadratic function and hence go to infinity as $|x| \rightarrow \infty$ (i.e. $V(x, i) \geq c_{3} x^{2}+c_{4}, i=0$, $\left.1, c_{3}>0\right)$. A detailed proof of these properties is available in [6]. For convenience, we report here a sketch of this proof.

a) The positivity of $H(x)>0$ for all $x$ is obtained directly from the HJB equations (10) and (11) (considered in the classical sense between the switching levels) which allow to prove that for all $x$ where $V(x, 1)$ is differentiable $H_{x}(x):=d H / d x \geq-\left(\left(q_{u p}+\right.\right.$ $\left.\left.q_{d}(0)\right) / d\right) H(x)$.In addition, from (11), using the quadratic lower bound on $V(x, 0)$ (property (d)), it is possible to show that there exists a sequence $x_{k}$, with $x_{k} \rightarrow-\infty$ as $k \rightarrow \infty$, where $H\left(x_{k}\right)>0$. These two results imply that $H(x)>0$ for all $x$.

b) To show the continuous differentiability of $V(x, 1)$ also on the switching levels, we use a procedure similar to the one adopted for the same purpose in [9], ch. 3.3, which applies under Condition (i) or (ii) of the theorem.

c) As for the quadratic bound on $|V(x, i)|, i=0,1$, this can be shown using a result in [8] according to which if $\tau$ denotes the time necessary for going from any given state $\left(x^{\prime}, i\right)$ to another state $(x, j)$ (with $x>x^{\prime}$ ) by working at a rate $U_{k}>d$, then $\tau$ is such that $E\left[\tau^{\ell}\right] \leq c_{a}+c_{b}\left|x-x^{\prime}\right|^{\ell}$ (where $\ell=1,2, \ldots$ ).

d) The quadratic lower bound on $|V(x, i)|, i=0,1$ can be proved in a way similar (but simpler) to the one used for the quadratic upper bound in item (c) above.

Then, the proof of Theorem 2 is concluded by showing that the policy $u(x)$ satisfying the HJB equations (10), (11) (in the classical sense) with the aforementioned properties (a)-(d) of the functions $V(x, i)$, is policy (6). Now, from (10), we immediately have

$$
u^{*}(x)=\arg \min _{u \in[0, \mu]}\left[u V_{x}(x, 1)+q_{d}(u) H(x)\right] .
$$

Since $H(x)>0$ for all $x$ :

- $u^{*}(x)=0$ if $V_{x}(x, 1)>0$;

- $u^{*}(x)=U_{i}$ for some $i$ if $V_{x}(x, 1)<0$;

- $u^{*}(x) \in\left[0, U_{1}\right]$ if $V_{x}(x, 1)=0$.

Let $Z_{g}:=\inf \left\{x: V_{x}(y, 1)<0 \forall y<x, V_{x}(x, 1)=\right.$ 0 and there exists $\left.\epsilon>0: V_{x}(y, 1)>0 \forall y \in(x, x+\epsilon)\right\}$ (this $Z_{g}$ exists thanks to the fact that $V(x, 1)$ is continuously differentiable and goes to infinity as $|x| \rightarrow \infty)$. Based on (24), the region $\left\{x>Z_{g}\right\}$ is transient (the optimal control is 0 over a non-zero interval $\left(Z_{g}, Z_{g}+\epsilon\right)$ and eventually the buffer will drop below $Z_{g}$ ). To complete the proof that the policy in (6) is candidate to solve the HJB equations, we need to further develop the second item above, that is, which $U_{i}$ is optimal when $x<Z_{g}$ (where $V_{x}(x, 1)<0$ ). From (24) it can be seen that a rate $U_{k}$ is optimal at $x<Z_{g}$ if and only if

$$
V_{x}(x, 1) U_{k}+H(x) q_{k} \leq V_{x}(x, 1) U_{i}+H(x) q_{i}
$$

for all $i$, i.e. if and only if

$$
V_{x}(x, 1) \leq-H(x) \phi_{i k}
$$

for all $i<k$ (where $\phi_{i k}$ has been defined in (7)) and

$$
V_{x}(x, 1) \geq-H(x) \phi_{k i}
$$

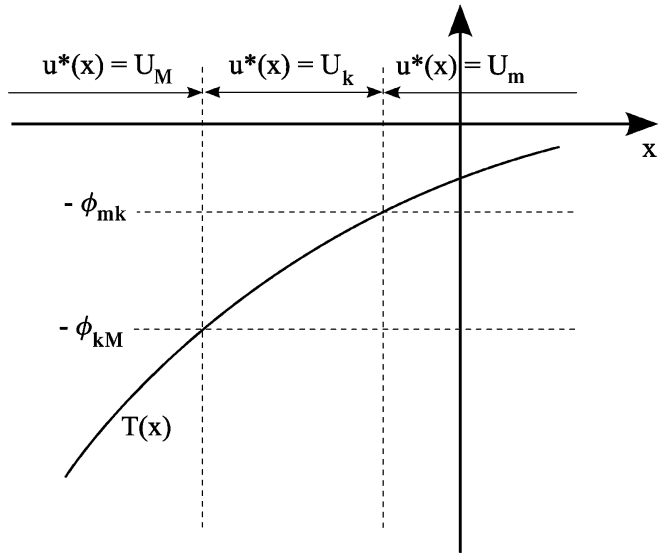

Fig. 1. Graphical representation of (28).

for all $i>k$. Exploiting the fact that $H(x)>0$ for all $x$, and introducing the function

$$
T(x):=\frac{V_{x}(x, 1)}{H(x)}
$$

the relations above will result in the following conditions:

- $U_{1}$ is optimal at $x$ if and only if

$$
T(x) \geq \max _{i>1}\left(-\phi_{1 i}\right)
$$

- $U_{k}, k=2, \ldots, N-1$ is optimal at $x$ if and only if

$$
\min _{i<k}\left(-\phi_{i k}\right) \geq T(x) \geq \max _{i>k}\left(-\phi_{k i}\right)
$$

- $U_{N}$ is optimal at $x$ if and only if

$$
T(x) \leq \min _{i<N}\left(-\phi_{i N}\right)
$$

Fig. 1 helps to figure out what is happening. In the figure $-\phi_{m k}=$ $\min _{i<k}\left(-\phi_{i k}\right)$ and $-\phi_{k M}=\max _{i>k}\left(-\phi_{k i}\right)$. According to (28), if $\min _{i<k}\left(-\phi_{i k}\right)<\max _{i>k}\left(-\phi_{k i}\right)$ for some $k, U_{k}$ can never be optimal. It remains to show that (27)-(29) result in the sequence $\mathcal{S}$ defined in Section III. This can be proved as follows: thanks to Lemma 1, we know that the intervals $\left[-\phi_{k M},-\phi_{m k}\right]$ provide a partition of the real axis. So, to conclude the proof, it is enough to show, for all $x \leq Z_{g}$, the following facts: 1) $T(x)$ is a continuous and negative function, with $T\left(Z_{g}\right)=0$;2) $T(x)$ intersects each $m_{j}$, for $j=2, \ldots, L^{\prime}$, at most once and, 3) the last element $i_{L} \in \mathcal{S}$ is the first element in $\mathcal{S}^{\prime}$ such that $\Delta U_{i_{L}, i_{L+1}} \geq 0$.

1) Continuity and Negativity of $T(x)$, With $T\left(Z_{g}\right)=0$ : Since $H(x)>0$ for all $x, V_{x}(x, 1)<0$ for all $x<Z_{g}$ and $V_{x}\left(Z_{g}, 1\right)=0$, it immediately follows that $T(x)<0$ for all $x<Z_{g}$ and $T\left(Z_{g}\right)=0$. In addition, the continuous differentiability of $V(x, 0)$ and $V(x, 1)$ implies that $V_{x}(x, 1)$ and $H(x)$ are continuous functions for all $x$. Hence $T(x)$ is continuous for all $x$.

2) Unique Intersection of $T(x)$ With the $m_{j}$ 's: Now we show that $T(x)$ can intersect a given $m_{j}, j=2, \ldots, L^{\prime}$, at most once. This is done by contradiction. Assume that there are two (or more) intersections of $T(x)$ with a given $m_{j}$ and, to simplify notation, let $i_{j}=k$ and $i_{j-1}=l$, with $m_{j}=-\phi_{l k}$ and $U_{k}>U_{l}$ (see Fig. 2). Notice that this is the most general case being $T\left(Z_{g}\right)=0$ and $T(x)<0$ for all $x<Z_{g}$, hence $T(x)$ is certainly increasing in a neighbor on the left of $Z_{g}$. So the first time (starting from $Z_{g}$ and going left) we would observe the intersection of $T(x)$ with a level $m_{j}$ already met, it must be of the type reported in Fig. 2, where $T\left(X_{k 1}\right)=T\left(X_{k 2}\right)=m_{j}$. 


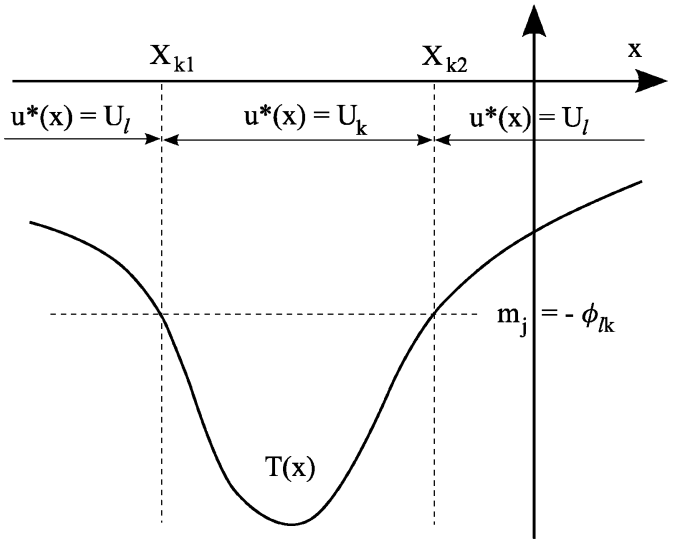

Fig. 2. Case of multiple intersections between $T(x)$ and a given $m_{j}$.

Now, due to the continuity of $V_{x}(x, 1)$, from the HJB equation (10) at $X_{k 1}$ and at $X_{k 2}$ (i.e. from $\left.(d V(x, 1) / d x)\right|_{x=X_{k_{i}}^{-}}=$ $\left.\left.(d V(x, 1) / d x)\right|_{x=X_{k_{i}}^{+}}, i=1,2\right)$ we obtain

$$
H\left(X_{k_{1}}\right)=-\frac{\left(J^{*}-g\left(X_{k_{1}}\right)\right)\left(U_{l}-U_{k}\right)}{B_{k l}}
$$

and

$$
H\left(X_{k_{2}}\right)=-\frac{\left(J^{*}-g\left(X_{k_{2}}\right)\right)\left(U_{k}-U_{l}\right)}{B_{l k}}
$$

where $B_{l k}=q_{k}\left(U_{l}-d\right)-q_{l}\left(U_{k}-d\right)$. Notice that $B_{k l}=-B_{l k}$, and that it can have any sign even if $U_{k}>U_{l}$. Also, $B_{k l} \neq 0$ since $H(x)$ is a continuous function, and at least one between $J^{*}-g\left(X_{k_{2}}\right)$ and $J^{*}-g\left(X_{k_{1}}\right)$ must be different from 0 . This depends on the fact that $X_{k 1}<X_{k 2}<Z_{g}$ and, as shown afterwards, $J^{*}>g\left(Z_{g}\right)$. So, to be $J^{*}-g\left(X_{k_{2}}\right)=J^{*}-g\left(X_{k_{1}}\right)=0$ it must be $X_{k 1}<X_{k 2}<0$. But then it is not possible that $-c_{m} X_{k 1}=-c_{m} X_{k 2}=J^{*}$.

Now, the fact that $B_{k l}=-B_{l k} \neq 0$, together with (30), (31) and the fact that $H(x)>0$ for all $x$, implies that $J^{*}-g\left(X_{k_{1}}\right)$ and $J^{*}-g\left(X_{k_{2}}\right)$ have the same sign, positive if $B_{k l}>0$ and negative otherwise. Denote by $R_{s}$ the set of all $x \leq Z_{g}$ where the $\operatorname{cost} g(x)$ is smaller than $J^{*}$. Then we have $R_{s}=\left(x_{s}, Z_{g}\right)$, where $x_{s}<0$ is such that $g\left(x_{s}\right)=$ $-c_{m} x_{s} \equiv J^{*}$. In fact, as mentioned above, from (10), with $x=Z_{g}$, it follows that (thanks to the positivity of $H\left(Z_{g}\right)$ ), $J^{*}>c_{p} Z_{g}$, i.e., $J^{*}>g(x)$ for all $x \in\left(0, Z_{g}\right)$. For this reason $R_{s}$ ends at $Z_{g}$.

Now, assume first that $B_{k l}<0$ (later we will see the other case). This implies that $X_{k 2}<x_{s}$ (and $X_{k 1}<x_{s}$ as well). A direct computation allows to show that

$$
T_{k 1}^{\prime}:=\lim _{x \rightarrow X_{k 1}^{-}} T^{\prime}(x)=\frac{1}{\left[H\left(X_{k 1}\right)\right]^{2}} \frac{N_{0}+N_{1} H\left(X_{k 1}\right)}{d\left(U_{l}-d\right)}
$$

where $N_{0}=U_{l}\left(J^{*}-g\left(X_{k 1}\right)\right)^{2}$ and $N_{1}=\left(J^{*}-g\left(X_{k 1}\right)\right) \Delta_{l}+$ $c_{m} d\left(U_{l}-d\right)$. From (32), using (30), it is possible to obtain the following expression for the left derivative of $T(x)$ as $x \rightarrow X_{k 1}$ :

$$
T_{k 1}^{\prime}=\frac{J_{k 1}}{\left[H\left(X_{k 1}\right)\right]^{2}} \frac{J_{k 1} \Delta U_{k l}+c_{m} d\left(U_{k}-U_{l}\right)}{d B_{k l}}
$$

where $J_{k 1}:=J^{*}-g\left(X_{k 1}\right)$ is a negative quantity, being $X_{k 1}<x_{s}$. Similarly, the left derivative of $T(x)$ as $x \rightarrow X_{k 2}$ can be expressed as

$$
T_{k 2}^{\prime}=\frac{J_{k 2}}{\left[H\left(X_{k 2}\right)\right]^{2}} \frac{J_{k 2} \Delta U_{k l}+c_{m} d\left(U_{k}-U_{l}\right)}{d B_{k l}}
$$

where $J_{k 2}:=J^{*}-g\left(X_{k 2}\right)$, and $0>J_{k 2}>J_{k 1}$, being $X_{k 1}<X_{k 2}<$ $x_{s}$. It must be $T_{k 1}^{\prime}<0$ and $T_{k 2}^{\prime}>0$ (see Fig. 2). Now, if $\Delta U_{k l} \leq 0$, it is clear that both $T_{k 1}^{\prime}$ and $T_{k 2}^{\prime}$ are positive, and this would exclude the possibility of a double intersection. Assume then that $\Delta U_{k l}>0$ (notice that $\Delta U_{k l}$ can take any sign even if $B_{k l}<0$ ). In this case, as explained below (see Section IV-3 and Lemma 2), the last element of the optimal sequence is some $U_{i}$, with $i \geq k>l$, so the function $T(x)$ should intersect again $m_{j}$ at some $X_{k 0}<X_{k 1}$ with a positive left derivative $T_{k 0}^{\prime}$ given by

$$
T_{k 0}^{\prime}=\frac{J_{k 0}}{\left[H\left(X_{k 0}\right)\right]^{2}} \frac{J_{k 0} \Delta U_{k l}+c_{m} d\left(U_{k}-U_{l}\right)}{d B_{k l}} .
$$

Now, $J_{k 1}>J_{k 0}$, hence we have that if $T_{k 1}^{\prime}$ is negative, negative must also be $T_{k 0}^{\prime}$. Hence, the possibility of a multiple intersection is excluded also in this case.

Assume now that $B_{k l}>0$. From (30) and (31), it follows that $I_{k}:=$ $\left(X_{k 1}, X_{k 2}\right) \subset R_{s}$. In this case, either $X_{k 1}<0$ or $X_{k 1} \geq 0$.

If $X_{k 1} \geq 0$ (hence also $X_{k 2}>0$ ), the expression of the left derivative of $T(x)$ as $x \rightarrow X_{k 2}$ can be expressed similarly to (34) and is given by

$$
T_{k 2}^{\prime}=\frac{J_{k 2}}{\left[H\left(X_{k 2}\right)\right]^{2}} \frac{J_{k 2} \Delta U_{k l}-c_{p} d\left(U_{k}-U_{l}\right)}{d B_{k l}} .
$$

To have, as required, a positive $T_{k 2}^{\prime}$, it must be $\Delta U_{k l}>0$ (being in this case $J_{k 2}>0$ and $B_{k l}>0$ ). Since $J_{k 2}<J_{k 1}$ in this case, it follows that $J_{k 2} \Delta U_{k l}<J_{k 1} \Delta U_{k l}$, that is, being

$$
T_{k 1}^{\prime}=\frac{J_{k 1}}{\left[H\left(X_{k 1}\right)\right]^{2}} \frac{J_{k 1} \Delta U_{k l}-c_{p} d\left(U_{k}-U_{l}\right)}{d B_{k l}}
$$

also $T_{k 1}^{\prime}$ should be positive, in contrast with the behavior assumed (see Fig. 2).

If, on the other hand, $X_{k 1}<0$, from (33), to have, as required, a negative $T_{k 1}^{\prime}$, it should be $\Delta U_{k l}<0$. But then also $T_{k 2}^{\prime}$ will be negative, both if $X_{k 2} \geq 0$ (just consider (35)), both if $X_{k 2}<0$ (being in this case $J_{k 1}<J_{k 2}$, hence $J_{k 2} \Delta U_{k l}<J_{k 1} \Delta U_{k l}$ and comparing (34) with (33)). Hence, also if $B_{k l}>0$, the possibility of a multiple intersection is excluded.

3) Determination of the Last Element of $\mathcal{S}$ : To prove that the last element of $\mathcal{S}$ is the first $L \geq \ell$ in $\mathcal{S}^{\prime}$ such that $\Delta U_{i_{L}, i_{L+1}} \geq 0$, we compute $T_{\infty}:=\lim _{x \rightarrow-\infty} T(x)$ and show that it is a finite quantity with $T_{\infty} \in\left[M_{L}, m_{L}\right]$.

As for the computation of $T_{\infty}$, we proceed as follows. Let $U_{k}$ be the value of $u(x)$ on a certain interval $I_{k}:=\left(X_{k_{1}}, X_{k 2}\right)$ (notice that this corresponds to have $-\phi_{k M}<T(x)<-\phi_{m k}$ for all $x \in I_{k}$, for some $m<k<M$, see Fig. 1). The solutions $V(x, 0)$ and $V(x, 1)$ to the HJB equations in $I_{k}$ can then be obtained and an explicit expression is available in [6]. From this explicit expression it is possible to obtain

$$
V_{x}(x, 1)=A_{g} x+B_{g} e^{-\alpha_{k}\left(x-X_{k 2}\right)}+C_{g}
$$

and

$$
H(x)=A_{h} x+B_{h} e^{-\alpha_{k}\left(x-X_{k 2}\right)}+C_{h}
$$

where $A_{g}, B_{g}, C_{g}, A_{h}, B_{h}$ and $C_{h}$ are suitable constants. Since $|V(x, 0)|$ and $|V(x, 1)|$ are, as mentioned, bounded by a quadratic function for all $x$, it is clear that $B_{g}=0$ and $B_{h}=0$ if we are considering the most negative interval (i.e. the one with $X_{k 1}=-\infty$ ). From this, it is possible to see that

$$
T_{\infty}:=\lim _{x \rightarrow-\infty} T(x)=\lim _{x \rightarrow-\infty} \frac{A_{g} x+C_{g}}{A_{h} x+C_{h}}=\frac{A_{g}}{A_{h}} .
$$


Substituting the analytical expression of $A_{g}$ and $A_{h}$ (which can be obtained from the explicit expressions of $V(x, 0)$ and $V(x, 1)$ in [6]) and simplifying, allows to show that

$$
T_{\infty}=\frac{A_{g}}{A_{h}}=-\frac{q_{u p}+q_{k}}{U_{k}}<0 .
$$

Let $i_{R}, R \leq L^{\prime}$, be the last element of $\mathcal{S}^{\prime}$ used by the optimal policy and let for convenience $m_{L^{\prime}+1}=-\infty$. It must be

$$
m_{R+1} \leq T_{\infty}<m_{R}
$$

i.e., using the expression of $T_{\infty}$ in (39)

$$
m_{R+1} \leq-\frac{q_{u p}+q_{i_{R}}}{U_{i_{R}}}<m_{R}
$$

From the expression of $m_{R}$ and $m_{R+1}$ (see (13)), it is possible to show that this condition corresponds to $\Delta U_{i_{R-1}, i_{R}}<0$ and $\Delta U_{i_{R}, i_{R+1}} \geq 0$. Since, according to Lemma $2, \Delta U_{i_{k}, i_{k+1}} \geq 0$ implies $\Delta U_{i_{k+1}, i_{k+2}} \geq 0$, this means that all the elements $i_{k}$, with $k<R$, are such that $\Delta U_{i_{k}, i_{k+1}}<0$ while all the $i_{k}$, with $k>R$, are such that $\Delta U_{i_{k}, i_{k+1}} \geq 0$. This allows to conclude that, when constructing the sequence $\mathcal{S}$ from $\mathcal{S}^{\prime}$, we have to stop at $L$, which is in fact the first element of $\mathcal{S}^{\prime}$ such that $\Delta U_{i_{L}, i_{L+1}} \geq 0$.

Now, if $U_{i}>d$ for all $i$ (Condition (i) of the theorem), the proof is complete, with $Z^{*}:=X_{\ell}^{*}=Z_{g}$. On the contrary, if $U_{i}<d$ for some $i$ (but Condition (ii) of the theorem holds), we have proved so far the optimality of a policy which uses some $U_{i_{j}}<d$ for $x \in\left(Z^{*}, Z_{g}\right)$. However, if in a given region it is optimal to apply a control $U_{i}<d$, this region is transient and we have decided (for simplicity of notation in (6)) to replace all these $U_{i}$ with $u=0$. This does not influence the steady state average performance index considered in this technical note but only the transient behavior.

\section{CONCLUSION}

The problem of minimizing a long term average expected backlog/inventory cost for a manufacturing system comprising a machine characterized by a Markovian, production dependent failure rate process has been considered in this technical note. The dependence of the failure rate on the production rate has been described through a piecewise function which can be thought of as the discrete approximation of a continuous failure rate function. The discretization step can be selected to obtain the desired degree of approximation. The structure of the optimal policy has been given in the technical note: even if under the discrete approximation considered, this policy confirms several analytical findings and conjectures reported in the literature.

\section{REFERENCES}

[1] T. Bielecki and P. R. Kumar, "Optimality of zero-inventory policies for unreliable manufacturing systems," Oper. Res., vol. 36, no. 4, pp. 532-541, 1988.

[2] S. Gershwin, Manufacturing Systems Engineering. Englewood Cliffs, NJ: Prentice Hall, 1994.

[3] J. Q. Hu, P. Vakili, and G. X. Yu, "Optimality of hedging point policies in the production control of failure-prone manufacturing systems," IEEE Trans. Autom. Control, vol. AC-39, no. 9, pp. 1875-1880, Sep. 1994.

[4] G. Liberopoulos and M. Caramanis, "Production control of manufacturing systems with production-rate dependent failure rates," IEEE Trans. Autom. Control, vol. AC-39, no. 4, pp. 889-895, Apr. 1994.

[5] F. Martinelli, "Optimality of a two threshold feedback policy for the control of a production dependent, failure prone manufacturing system," IEEE Trans. Autom. Control, vol. AC-52, no. 10, pp. 1937-1942, Oct. 2007.
[6] F. Martinelli, "Manufacturing Systems With a Production Dependent Failure Rate: Structure of Optimality," Dip. di Informatica, Sistemi e Produzione, Università di Roma “Tor Vergata", Roma, Italy, Tech. Rep. RR-10.84, Jun. 2010.

[7] S. Sethi, H. Zhang, and Q. Zhang, Average-Cost Control of Stochastic Manufacturing Systems. New York: Springer, 2005.

[8] S. Sethi, W. Suo, M. I. Taksar, and Q. Zhang, "Optimal production planning in a stochastic manufacturing system with long-run average cost," J. Optim. Theory Appl., vol. 92, no. 1, pp. 161-188, Jan. 1994.

[9] S. Sethi and Q. Zhang, Hierarchical Decision Making in Stochastic Manufacturing Systems. Boston, MA: Birkhauser, 1994.

[10] M. Veatch and M. Caramanis, "Optimal average cost manufacturing flow controllers: Convexity and differentiability," IEEE Trans. Autom. Control, vol. AC-44, no. 4, pp. 779-783, Apr. 1999.

\section{Delay-Independent Minimum Dwell Time for Exponential Stability of Uncertain Switched Delay Systems}

\author{
Wu-Hua Chen and Wei Xing Zheng, Senior Member, IEEE
}

Abstract-In this technical note, the problem of delay-independent minimum dwell time for exponential stability of uncertain switched delay systems is considered. Piecewise time-varying Lyapunov functionals/functions which are decreasing at switching times by construction are introduced to investigate exponential stability of switched delay systems with constant or time-varying delays. This type of delicately constructed Lyapunov functionals/functions can efficiently eliminate the "jump" phenomena of adjacent Lyapunov functionals/functions at switching times without imposing any restriction on the sizes of time-delays. By applying this type of Lyapunov functionals/functions, it is shown that if each subsystem is delay-independently exponentially stable, then under some conditions there exists a delay-independent minimum dwell time in the sense that the switched delay system with such minimum dwell time is exponentially stable irrespective of the sizes of the time-delays. Two numerical examples are provided to demonstrate the efficiency of the proposed approach.

Index Terms-Delay-independent, exponential stability, minimum dwell time, switched systems, time delay.

\section{INTRODUCTION}

Switched systems are a special class of hybrid systems, which consist of a family of distinct active subsystems subject to a certain switching rule which chooses one of them being active during a certain time. These systems arise when modeling dynamical systems which exhibit switching among several subsystems due to jumping parameters or changing environmental factors. Some examples of switched control systems can be found in [4], [5], [10].

Manuscript received July 12, 2009; January 31, 2010, and May 06, 2010; accepted June 08, 2010. Date of publication June 28, 2010; date of current version October 06, 2010. This work was supported in part by the National Natural Science Foundation of China under Grant 60864002, and in part by a research grant from the Australian Research Council. Recommended by Associate Editor H. Fujioka.

W.-H. Chen is with the College of Mathematics and Information Science, Guangxi University, Nanning, Guangxi 530004, China. Part of this work was done when he was with the School of Computing and Mathematics, University of Western Sydney, Penrith South DC NSW 1797, Australia (e-mail: wuhua_chen2002@yahoo.com.cn).

W. X. Zheng is with the School of Computing and Mathematics, University of Western Sydney, Penrith South DC NSW 1797, Australia (e-mail: w.zheng@uws.edu.au).

Digital Object Identifier 10.1109/TAC.2010.2054845 